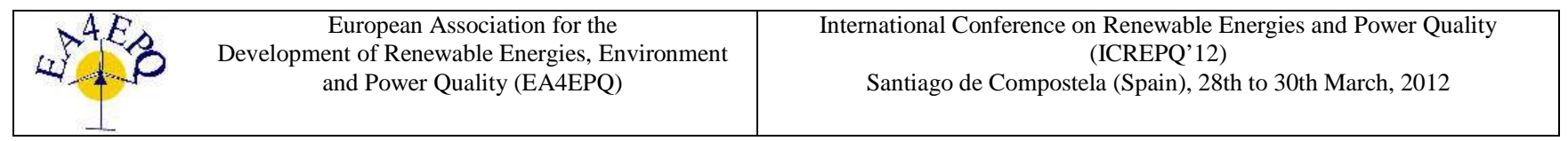

\title{
Grid-connected PV-systems Joule design using Evolutionary Strategies
}

\author{
Daniel Gómez-Lorente ${ }^{1}$, Enrique Alameda-Hernández ${ }^{1}$, F. Aznar Dols ${ }^{1}$, M.J. Mercado-Vargas ${ }^{1}$ and A. Espín \\ Estrella ${ }^{1}$ \\ ${ }^{1}$ Electrical Engineering Section, Department of Civil Engineering \\ E.T.S.I.C.C.P, Granada University \\ Campus of Fuentenueva 18071 Granada (Spain) \\ Phone/Fax number: +0034 958 249435/+0034 958 246138, e-mail: dglorente@ugr.es
}

\begin{abstract}
This paper shows how to compute the optimal distribution of the main elements of a photovoltaic (PV) plant with trackers connected to the network, to reduce some of the losses that occur in these facilities. In particular, the objective function of the proposed optimization process is the minimization of Joule losses that occur in electric conductors. To solve this problem, genetic algorithms (GAs), implemented in Matlab, have been used. Specifically the steady-state GA and the generational GA, which from random initial solutions, possibly far from optimal, will evolve into solutions that are increasingly closer to the desired optimum. Results have been very satisfactory, since all proposed examples have reduced by more than $50 \%$ electrical losses originated by not optimally designing the PV plant.
\end{abstract}

\section{Key words}

Photovoltaic plants, Joule losses, Genetic algorithms, Numerical optimization.

\section{Introduction}

It is true that installation costs of photovoltaic grid connected systems (PVGCSs) are decreasing due to the standardization of technology and rising demand among other factors, but on the contrary, the premiums for the sale of electricity and subsidies for the initial installation of PVGCSs are also lower due to the inability of governments to maintain the high initial aid, therefore, any project of a PVGCSs which can optimize the energy output and reduce the cost of installation should be investigated and acted upon.

On one hand, methods of sizing stand-alone photovoltaic (PV) systems [1] and PVGCSs with trackers [2] have been presented. On the other hand, studies have been devoted to increase the performance of PVGCSs, choosing and sizing inverters to take full advantage of the power generated by them [3-5] and how this sizing factor between the inverters and PVGCSs is affected by temperature and location of land where the PV plant is [6].
In a PV plant there are many electrical losses: mismatch losses, soil losses, angular losses, temperature losses, selfshading losses, losses by inverter efficiency and Joule looses, and the relevance of each one on the others varies according to the characteristics of the PV system. In this paper we have decided to start the optimization problem of PVGCSs with trackers considering just Joule losses. So, we find, within the different variables that define the plant, the optimums that minimize Joule losses and increase the generated power. These variables are the distribution of the modules within the tracking structure, the choice of a particular PV module, the choice of a particular inverter and the distribution of trackers in the specific field. We will not consider among these variables rated power, since it has been shown in the studies mentioned above which the relationship between rated power of the inverter and rated power of the PV generator should be.

To optimize the design of PV systems many artificial intelligence techniques have been used [7]. In this work, in particular, this optimization has been performed using genetic algorithms (GAs) [8], which, from random initial solutions, far from optimal, will evolve into more advanced solutions close to the desired optimum. This will be done using the Matlab programming language as the tool to build the GA. To the best of our knowledge, GAs have not yet been applied to optimizing the design of PVGCs with solar tracking.

GAs were envisaged by Holland [9] in the 1970s as an algorithmic concept based on a Darwinian-type survivalof-the-fittest strategy with sexual reproduction, where stronger individuals in the population have a higher chance of creating an offspring. GA's are inspired by the way living organisms are adapted to the harsh realities of life in a hostile world, i.e., by evolution and inheritance. The algorithm imitates in the process the evolution of population by selecting only fit individuals for reproduction. Therefore, a GA is an optimum search technique based on the concepts of natural selection and survival of the fittest. It works with a fixed-size 
population of possible solutions of a problem, called individuals, which are evolving in time. A GA utilizes three principal genetic operators: selection, crossover, and mutation [10-11].

Compared to conventional optimization methods, such as dynamic programming and gradient techniques, the GA has the ability to attain the global optimum with relative computational simplicity. GAs have been applied to the design of large power distribution systems [12] because of their ability to handle complex problems with linear or non-linear cost functions both, accurately and efficiently. In the proposed method, GAs are selected because they have shown to be highly applicable to cases of large nonlinear systems, where the location of the global optimum is a difficult task. Two variants of the GA have been studied (steady-state GA, and generational GA [13]) and the efficiency of each type has been verified given three problems which consist of areas with different dimensions, in which we tried to distribute and to choose the elements that compose the PV plant in a way such that fewer possible electric losses are generated.

Based on the paper's results that show the feasibility of GAs to address PVGCSs optimization problems, all possible losses can be included in the future with the goal of increasing the plant overall efficiency. It is enough to add terms to the cost function, and to design this cost function to maximize or minimize the desired criteria. The rest of the paper is organized as follows: Section 2 gives a description of the evolutionary strategies used. Section 3 gives a description of the proposed problem and how can be solved with these evolutionary strategies. Section 4 discusses the experimental framework and presents the analysis of results. Finally, in Section 5 we summarize our conclusions.

\section{Proposed evolutionary strategies}

Two evolutionary strategies to establish a comparative performance study have been chosen.

\section{A. A steady-state genetic algorithm}

In a steady-state GA, only a few individuals are inserted in the population substituting some of the parents - usually one or two - in each generation (one in this case substituting the worst individual). Duplicates are not allowed in the population, so they are avoided in the initial population and also during the insertion in the evolving population along the execution of the algorithm. A simple crossover [14] is used. Selection is done by tournament selection, according to the selection probability of the individuals. The crossover probability is 1.0.

\section{B. A generational genetic algorithm}

Unlike the first one, in the generational strategy, the parent population as a whole is substituted by their children. With elitism, the best individual of each generation is conserved and inserted directly in the next generation. A BLX- $\alpha$ with $\alpha=0.4$ [14] crossover is chosen. Selection is done by tournament according to the selection probability of the individuals. The crossover probability is 0.9 .

\section{Problem Statement}

The problem that arises is as follows. Given a rectangular field with specific dimensions and latitude, the algorithm will try to find the optimal configuration among the different possible configurations, so that the Joule losses in electrical conductors from trackers to inverters are minimal. Two-axis trackers have been chosen for the study as a situation in which PV modules operate closer to the nominal conditions, consequently, the algorithm considers the maximum tilt angle of the vertical axis for the calculation of the shadows length. Each configuration is a set of certain variables that define some aspects of the PV plant: the number of rows and columns of PV modules within the tracking structure, the value of the peak power of our PV modules, the intensity at the point of maximum power generated by the PV module, the dimensions of the PV module, and the nominal and maximum voltage input to the inverter. Each solution to our problem (chromosome) consists of eight independent parts (genes) as Fig. 1 shows.

At the beginning of the algorithm the maximum and minimum values that these variables can take are specified, so that, in its search, the algorithm does not get out of these ranges and does not lead to completely inconsistent solutions. It is important to note that these ranges are input to the algorithm and they are fully adjustable at the beginning of the calculation. Thus, to solve our problem, the values found in the catalogs of the leading manufacturers of PV modules and inverters have been introduced, as limiting values for these ranges.

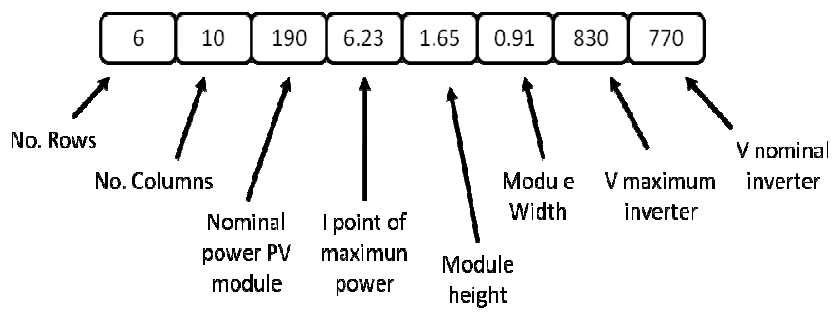

Fig. 1. Sample of a chromosome with eight genes for our genetic algorithm.

Moreover, some additional features have been introduced to determine the solutions that the algorithm provides, so that the relationships that exist between some of these variables are not broken. For example, if the algorithm in its search space is looking for higher power modules, this is conditioned upon the physical dimensions of these modules, which also increase in some proportion, and vice versa. Furthermore, the fitness function is scaled through ranking, in order to avoid premature domination of a possible superindividual and premature stagnation and also to benefit the evolution in the last generations.

First, the algorithm calculates the tracking surface area with the rows and columns number of PV modules on the tracking structure, and the PV modules size. With the area 
value and the land latitude the algorithm is able to obtain the maximum shadow length of the tracker and therefore to obtain the minimum distance between trackers to avoid shadows on the nearby trackers. In this way, the algorithm, while minimizing Joule losses, avoids selfshading looses.

The procedure following the algorithm to obtain the Joule losses cost function in electrical conductors that connect the solar trackers with inverters is as follows: first, from the open circuit voltage drops of PV modules (Voc) and the inverter's input voltage (Vcc) determine how many solar modules in series is capable of supporting the inverter and how many sets of modules in parallel have to be in the solar tracker. This will allow us to know the intensity flowing in these electrical conductors. On the other hand, to calculate the Joule losses in the conductor, we need to know the section of these cables. This section is obtained through the condition of maximum allowable voltage drop in the PV plant as:

$$
\Delta V=\frac{2 \cdot L \cdot P}{\mu \cdot s \cdot V}
$$

Once we have the intensity and the section of the conductor through which this intensity will circulate, we can easily calculate the Joule power losses in that conductor as:

$$
P=2 \cdot R \cdot I^{2}
$$

Each configuration is going to generate a different loss statement and therefore the losses for each of the possible configurations will give us the levels of competence of the chosen solution, which will allow the algorithm decide which solutions are best. The GA has been implemented in Matlab, and is composed of a main function from which four other auxiliary functions are called. Each of these auxiliary functions will be tasked to perform a function of the algorithm.

\section{Results and discussion}

The two described variants of the GA have been tested in three different scenarios when applied to the proposed problem of Joule losses reduction. Each scenario is determined by the characteristics of the field where the PV plant is located (see Table I).

Table I. Scenarios under consideration

\begin{tabular}{c|cc}
\hline Field & Dimension $X(\mathrm{~m})$ & Dimension $Y(\mathrm{~m})$ \\
\hline 1 & 250 & 150 \\
2 & 400 & 200 \\
3 & 120 & 90 \\
\hline
\end{tabular}

Location: Granada, Spain (latitude $37^{0} \mathrm{~N}$ )

Below is the response given by each one of the GA models mentioned above to the proposed problems. These variants were constraint to the design ranges defined in Table II.
Table II. Problem design ranges

\begin{tabular}{ccc}
\hline Parameter & Minimum & Maximum \\
\hline No Rows & 5 & 9 \\
$\mathrm{~N}^{\mathrm{o}}$ Columns & 8 & 14 \\
Module power (W) & 150 & 290 \\
I maximun power point (A) & 4,49 & 10,23 \\
Module height (m) & 1,324 & 2,000 \\
Module width (m) & 0,800 & 1,061 \\
Maximun inverter voltage (V) & 700 & 880 \\
Nominal inverter voltage (V) & 600 & 800 \\
\hline
\end{tabular}

Steady-state genetic algorithm. Simple crossover [14] has been used and the best results have been obtained using a mutation probability of $10 \%$. Fig. 2 shows the Joule losses, in percentage of nominal power, curves versus the number of iterations of the algorithm, for each of the three proposed sites. The best configuration obtained for each field can be seen in Table III.

Generational genetic algorithm. Best results have been obtained applying a crossover probability of $90 \%$ and a mutation probability of $20 \%$. The BLX- $\alpha$ with $\alpha=0.4$ [14] crossover has been chosen for this model. Fig. 3 shows the Joule losses curves. The best configuration obtained for each field can be seen in Table IV.

If we analyze the results, first it is observed how initial losses are independent of the method used, which otherwise is logical since the starting solution is totally random. The power losses over installed capacity are between $1,10 \%$ and $1,18 \%$ for the largest surface field, between $0,66 \%$ and $0,75 \%$ for the average surface field, and between $0,40 \%$ and $0,44 \%$ for the smallest of the studied fields, which tells us that if we made the design of the system in an arbitrary way, those are the losses we would get. After applying the optimization algorithms it can be seen how they have found solutions whose losses have decreased to $0.61 \%, 0.45 \%$ and $0.27 \%$ for the higher field, middle and lower respectively, which means a reduction losses around $50 \%$.

Moreover, if we compare the results provided by each of the methods used it can be seen how generational GA is significantly above steady-state GA in terms of energy losses and iterations needed. Thus, if on one hand, steadystate GA has found solutions whose losses are $0.67 \%$, $0.48 \%$ and $0.31 \%$, generational GA has reduced them to $0.61 \%, 0.46 \%$ and $0.27 \%$ for higher field, middle and lower respectively. In addition, the number of iterations required is also significantly lower in generational GA in which desired solution has been found at 500 iterations approximately, while steady-state GA needs around 1,500 iterations. 
Table III. Steady-state genetic algorithm results

\begin{tabular}{c|cccccccccc}
\hline Field & $\begin{array}{c}\text { No. } \\
\text { trackers }\end{array}$ & $\begin{array}{c}\text { Minimum } \\
\text { loss }(\%)\end{array}$ & Rows & Columns & Pow $(W)$ & $\operatorname{Imp}(A)$ & $Y(m)$ & $X(m)$ & $V_{\operatorname{maxinv}}(V)$ & $\begin{array}{c}V_{\text {nominv }} \\
(V)\end{array}$ \\
\hline 1 & 83 & 0,4786 & 6 & 8 & 158,38 & 5,52 & 1,67 & 0,89 & 879,88 & 739,46 \\
2 & 252 & 0,6658 & 5 & 9 & 151,06 & 5,24 & 1,71 & 0,88 & 811,79 & 765,55 \\
3 & 34 & 0,3103 & 6 & 8 & 169,21 & 5,81 & 1,33 & 1,06 & 878,57 & 765,32 \\
\hline
\end{tabular}

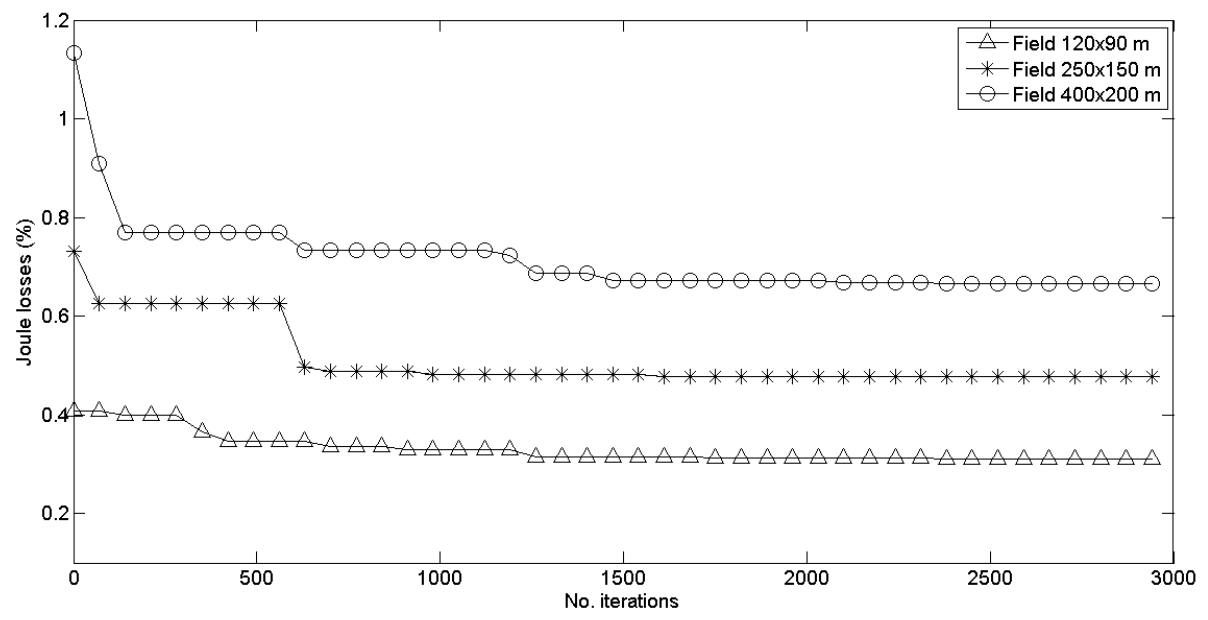

Fig. 2. Steady-state genetic algorithm simulation.

Table IV. Generational genetic algorithm results

\begin{tabular}{c|cccccccccc}
\hline Field & $\begin{array}{c}\text { No. } \\
\text { trackers }\end{array}$ & $\begin{array}{c}\text { Minimun } \\
\text { loss }(\%)\end{array}$ & Rows & Columns & Pow $(W)$ & $\operatorname{Imp}(A)$ & $Y(m)$ & $X(m)$ & $V_{\text {maxinv }}(V)$ & $V_{\text {nominv }}(V)$ \\
\hline 1 & 83 & 0,4587 & 5 & 8 & 169,54 & 5,15 & 1,97 & 0,99 & 831,65 & 760,44 \\
2 & 252 & 0,6118 & 5 & 9 & 162,70 & 5,22 & 1,75 & 0,93 & 876,88 & 651,38 \\
3 & 34 & 0,2714 & 5 & 8 & 176,81 & 5,20 & 1,52 & 0,97 & 849,98 & 695,06 \\
\hline
\end{tabular}

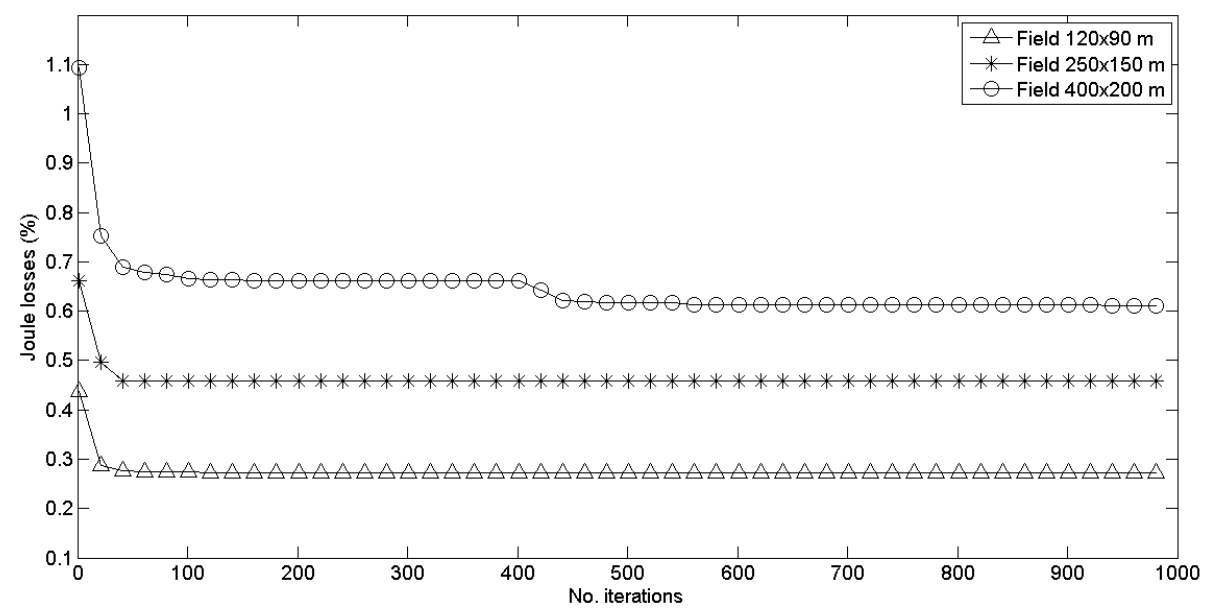

Fig. 3. Generational genetic algorithm simulation.

Finally, if we look at the end solutions (chromosomes) we find a tendency to opt for relatively small PV trackers where the number of rows and columns of PV modules is not very high, and how as expected, to choose settings which maximizes the input voltage to the inverter to thus associate the minimum number of PV modules in parallel and make that the intensity be as small as possible.

It is important to note that the electrical quantities $(\mathrm{P}, \mathrm{V}, \mathrm{I})$ were obtained as actual values of two decimals. If the sensitivity of these parameters is too high for the commercial election of a PV module or an inverter of these features, you can force the algorithm without any problem to take these results with a smaller number of decimal places or integers. This undoubtedly will affect the obtained loss solution.

\section{Conclusion}

In this work, the problem of optimizing the design of a solar PV plant with trackers has been addressed using a 
GA written in Matlab. The algorithm is able to find the optimal solution to minimize losses caused by Joule effect in electrical conductors that carry electrical current generated by PV modules located on the trackers to the distribution transformer.

Such optimization includes all the parameters that define the size and distribution of the components of a PV plant. Thus, given a field defined by its geometric characteristics and its latitude, the algorithm indicates the number of trackers that we should install, the PV modules that must be installed on these trackers and the type of inverter that must be chosen, to make the Joule losses minimal in the entire plant. Two variants or models of evolutionary algorithm: steady-state GA and generational GA have been used, and the obtained results compared in terms of electric power losses on electric power installed.

After an analysis of the results we can conclude that generational GA is the algorithm that provides better results for our problem, while from the point of view of the PV plant design, the best solutions tend to be configurations with small PV trackers where the number of rows and columns of PV modules is not very high.

Results have been very satisfactory, since all proposed examples have reduced by more than $50 \%$ electrical losses originated by not optimally placing the various elements that make up the plant without having to manufacture PV solar modules and solar inverters other than those currently on the market.

\section{Acknowledgement}

We wish to thank the Department of Computer Science and Artificial Intelligence of the University of Granada (DECSAI) for its invaluable contribution to this paper.

\section{References}

[1] Shrestha GB, Goel L. A study on optimal sizing of standalone photovoltaic stations. IEEE Transactions on Energy Conversion 1998; 13(4): 373-378.

[2] Hernández JC, Medina A, Jurado F. Optimal allocation and sizing for profitability and voltage enhancement of PV systems on feeders. Renewable Energy 2007; 32: 1768-1789.

[3] Burger B, Rüther R. Inverter sizing of grid-connected photovoltaic systems in the light of local solar resource distribution characteristics and temperature. Solar Energy 2006; 80: $32-45$.

[4] Velasco G, Guinjoan F, Piqué R, Conesa A, Negroni JJ. Inverter Power Sizing Considerations in Grid-Connected PV Systems. European Conference on Power Electronics and Applications 2007; 2310-2319.

[5] Macedo WN, Zilles R. Operational Results of GridConnected Photovoltaic System with Different Inverter's Sizing Factors (ISF). Progress In Photovoltaics: Research And Applications 2007; 15: 337-352.

[6] Mondol JD, Yohanis YG, Norton B. Optimal sizing of array and inverter for grid-connected photovoltaic systems. Solar Energy 2006; 80: 1517-1539.

[7] Mellit A, Kalogirou SA, Hontoria L, Shaari S. Artificial intelligence techniques for sizing photovoltaic systems: A review. Renewable Sustainable Energy Rewevs 2009; 13: 406419.
[8] Goldberg DE. Genetic algorithms in search, optimization, and machine learning. Reading, Mass.: Addison-Wesley, 1989.

[9] Holland JH. Adaptation in natural and artificial systems. Ann Arbor: University of Michigan Press, 1975.

[10] Kalogirou SA. Artificial intelligence for the modeling and control of combustion processes: a review. Progress in Energy and Combustion Science 2003; 29: 515-566.

[11] Kalogirou SA. Artificial Intelligence in energy and renewable energy systems. Nova Publisher 2007. 1-60021-2611.

[12] Ramirez-Rosado IJ, Bernal-Agustin JL. Genetic algorithms applied to the design of large power distribution systems. IEEE Transactions on Power Systems 1998; 13(2): 696-703.

[13] Greiner D, Winter G, Emperador JM. Optimizing frame structures by different strategies of genetic algorithms. Finite Elements in Analysis and Design 2001; 37: 381-402.

[14] Herrera F, Lozano M, Sánchez AM. A Taxonomy for the Crossover Operator for Real-Coded Genetic Algorithms: An Experimental Study. International Journal Of Intelligent Systems 2003; 18: 309-338. 